88

Concluderemo perlants che il depositarsi dei vapori su di una lasira, come risente l' influenza delle condensazioni, che per lo innanzi sono accadute diversamente sulle varie parti di quella lastra; cosi pure risente l'influenza delle pressioni e delle azioni chimiche che sopra alcuni punti di que'la lastra medesima sono antecedentemente accadute: e però si vede quanto moltiplici cagioni possono trovarsi dei fenomeni dipendenti da ineguale deposizione di vapori sopra una superficie senza che abbisogni ricorrere a forze nuove e straordinarie. Alle quali nel nostro caso ci sembra aver dimostrato doversi preferire la modificazione che una prima condensazione di vapori accaduta disugualmente su di una superficie imprime alla attitudine che ha questa superficie medesima a condensare nuovi vapori.

Doll. R. Rusch .

\title{
TEORIA di OHM SULLA PILA
}

\section{ARTIIOLO DEL, PROFESSORE CARLO MATTEUCCI}

Sono oramai sedici anni che Ohm pubblicò a Berlino un libro intitolato del circuito galvanico considerato matematicamente. La molta parte ipntetica di questo libro, la forma matematica con cui è redalto, le pochissime, se pur ve ne sono, esperienze proprie dell' autore, il fondarsi sulla forza elettro-motrice nel tempo in cui venia sviluppandosi la teoria chimira clella pila, infine la lingua in cui è scritlo, sono di certo le molte ragioni per cui è rimasto nell' oscurità e quasi generalmente ignorato. Pouillet fnndandosi sui principj dettati nel libro di Ohm ha, moltissimi anni dopo, pubblicata una lunga memoria in cui assai chiaramente sono esposti, $e$ in cui ha riferito un gran numero di espérienze che li confermano. In Ger. mania $e$ in Inghilterra, la teoria di Ohm è oggi generalmente adottata e non è più possibile ai meno istruiti nella Fisira l'ignorarne i fondamenti. 
Prendendo per guida le memorie di Pouillet in cui essi sono esposti con rhiarezza, e coll' appoggio dell' esperienza, cercheremo di comporne un arlicolo pei nostri Lettori.

Pouillet considera da prima il caso delle correnti termo-elettriche e dimostra sperimentalmente:

1. Che lintensità di una corrente è la stessa in tutti i punti del circuito che essa traversa; ch'è in ragione diretta della sezione del circuito, ed in ragione inversa della sua lunghezza .

Deduce da queste due leggi i rapporti di conducibilità di due diverse soslanze che compongono il circuito traversato da una corrente generaia dalla siessa sorgente. Delta $x$ la conduribilitz̀ di una di queste sostanze, $s^{\prime}$ la sezione, $l$ ' la lunghezza d'un circuito e chiamata 1 la conducibilita della sostanza a cui vuol riferirsi, $s$ la sua sezione, $l$ la sua lunghezza, le correnti avranno la stessa intensità, quando si avrà

$$
x=\frac{l^{\prime}}{l} \cdot \frac{s}{s^{\prime}} \text {. }
$$

Dunque l'intensita di una corrente, è in ragione diretta della sezione e della conducibiliti ed in ragione inversa della lunghezza del circuito.

Partendo dagli stessi principj sara farile di ridurre un circuiIn qualunque ad essere rappresentalo da un altrn circuito, di cui la conduribilità è data, facendo variare la sua lunghezza o la sua sezione.

E farile di comprendere come con una sorgente costante di eleuricità, qual può essere una coppia termo-eleltrica di cui le due saldature sono tenulc ad una costante differenza di temperatura, si può giungere a queste conclusioni, indipcndentemente da qualunque ipotesi sulla nalura della sorgente stessa, e sullo stato dell elettricita in corrente. Per Ohm il punto di partenza is la formola:

$$
I=\frac{E}{R}
$$

rhe signifira che l'intensità di una corrente è espressa da una frazione, di cui la forza eletm-motrice è il numeratore e la re- 
$9^{()}$

sistenza dell'intero circuito il denominatore. Presa $E$ costante, come lo ha fatto Pouillet nelle sue esperienze, i rapporti delle intensita di due correnti sono indipendenti dalla forza elettromotrice, $\mathrm{e}$ in ragione inversa delle resistenze intere dei circuiti che sono neressariamente in ragione diretta della loro lunghezza $e$ in raginne inversa delle sezioni e delle conducibilita.

Pouillet esamina il caso di un filo metallico di derivazione the unisce due punti diversi del circuito principale. $\mathbf{E}$ chiaro che questo filo di derivazione equivale ad un aumento di sezione della porzione compresa fra $\mathrm{i}$ due punti di derivazione " dell' intervallo di derivazione. Dai su esposti principj può trovarsi farilmente, qualunque sia questo filo di derivazione, a che corrisponda l'aumento di sezione dell' intervallo di derivazione e quindi la diminuzione di lunghezza corrispondente a questo aumento di sezione, conservata la sezione che aveva y,ima il filo. Si deducono da queste semplici considerazioni tutte le legri delle correnti derivate e le variazioni che avvengono sell'intensita della corrente principale per la derivazione, le yuali coincidono con quelle che l' esperienza aveva date.

Ne segue pure, che mentre in un circuito termoelettrico romposto di fili di diverse sezinni, la forza elementare della corrente è la stessa in tutti i punti, le correnti derivate da eguali intervalli di Jerivazinne sono assai diverse.

Pouillet è egualmente condotto a determinare la disposizione la più conveniente rhe deve adottarsi pel moltiplicatore, serondo la natura diversa del circuito elettro-motore. 11 filo del moltiplicatore è un filo aggiunto al circuito; è un aumento di resistenza alla corrente che l' indebolisce per conseguenza d' intensita. Questo flo aggiunto però, essendo ripiegato a più giri intorno all' ago, ripele ed accresre cosi la sua azione sull' ago stesso. Se il circuito primitivo è corto, molto grosso, luon condutiore, il filo del Galvanometro aggiunto, se non è grosso. poco lungo e bunn condutiore, accrescerà d'assai la resistenza alla corrente e non si guadagnerà di sensibilità nell' istrumento col numero dei giri. Se al contrario il circuito primitivo presenta 
nna grande resistenza, come nel caso della coppia idro-elettrica, in cui una porzione del circuito è il liquido interposto ai due elementi della coppia, vi sarì allora vantaggio ad adoperare per filo aggiunto un filo metallico lungo e sottile, che mentre accresre di poco la resistenza totale del circuito, rende possibile di ripetere con molti giri l'azione della corrente nell' ago .

Pouillet partendo dalle medesime considerazioni dà la formula che rappresenta l'intensita della corrente di una pila, la quale nel caso che tutti gli elementi agiscano colla medesima intensità si riduce alla seguente, quando si indichi con $E$ la forza eleltro-motrice di ciascuna coppia, con $r$ la resistenza del circuito d'ognuna, con $n$ il numero delle coppie, ed $l$ la resistenza del filo interpolare;

$$
I=\frac{n E}{n r+l}
$$

Da questa si vede facilmente che se $l$ è piccolissimo in confronto di $n r$, la formola si ridure a

$$
I=\frac{n E}{n r}=\frac{E}{r}
$$

cioè l'intensita della corrente è la stessa di quella somministrata da uná coppia sola. Se poi l fosse assai grande. allora non si potreble più trascurare in confronto di $n r ;$ e quando 'quest'ultimo fosse piccolissimo e trascurabile in confronto di $l$ allora la furmola si ridurrebbe

$$
I=\frac{n E}{l}
$$

rioè l'intensità della corrente sarebhe proporzionale a $n$, ossia al numero delle coppie. Nel caso poi che nessuno dei due termini $" r$ e $l$ si possa trascurare, si vede che crescendo il numero dell' coppie, l'intensita diventa sempre maggiore. Se iudichiamo con $I^{\prime}$ l'intensità di una corrente quando $E^{\prime}$ è la forza elettro-motrice, $l$ ' la resistenza interpclare, ed $n$ ' il numero delle coppie 
92

$$
I=\frac{r E}{n r+l} \quad I^{\prime}=\frac{n^{\prime} E^{\prime}}{n^{\prime} r+l} \text { prendéndo } E=E^{\prime}
$$

si ha $\frac{I^{\prime}}{I}=\frac{n r+l}{n^{\prime} r+l} \cdot \frac{n^{\prime}}{n}$

il qual rapporto è sempre maggiore di 1.

Queste leggi che assai facilmente si deducono coll' esperienza per il caso delle pile termo-életıriche, si complirano per le pile idro-elettriche di elementi che l'esperienza sin qui non è riuscita a ben determinare. In una coppia idro-elettrica il circuito si compone dell' arco metallico, delle due lamine eterogenee e di una massa liquida interposta. Onde estendere alle pile idro-elettriche le leggi trovate per le termo-elettriche, conveniva determinare la resistenza dell' intero circuito, e ridurla come si è delto di sopra, in una certa lunghezza di un filo metallico conosciuto, di una data sezione. Pouillet aggiungendo al circuito metallico di una coppia idro-eletirica a forza rostante, lunghezze diverse di uno stesso filo di rame, ha potuto dedurre a qual lunghezza di qu esto stesso filo, equivaleva la resistenza dell'elemento intiero, cinè della massa liquida e della coppia. Chiamando $x$ la resistenza dell' elemento, se vi si aggiungon diverse lunghezze di filo interpolare, si trovano deviazioni diverse nell' ago della bussola dalle tangenti. Ammettendo che le intensità delle correnti idro-elettriche siano in ragione inversa delle lunghezze del cirruito, è facile di dedurre il valore di $x$, rhe ammessa questa ipotesi, risulta costante. Si giunge egualmente a provare, come per le sorgenti termoelettriche, che l'intensilà di una corrente idro-eleltrica $\grave{e}$ in ragion diretta della sezione e della conducibiliti, $e$ in ragione inversa della lunghezza totale, che in un circuito cosi misto, non è già la lunghezza apparente, ma quella delle diverse parti del circuito ridotte ad un filo di una stessa sezione e di una data conducibilitì.

Basla queslo ad intendere la differenza che passa fra due sorgenti, una idro-elettrica, e una termo-eleltrica, che in 
realtà si riduce ad una differenza d' inteusita. Ripontiamo qui due esperienze di Pouillet in prova di quesla asserzione. Una corrente termo-elettrica prodotta da un elemento bismulo e rame, con una differenza di temperatura di $1000^{\circ}$ fra le due saldature, da' 16." alla bussola a secco. La lunghezza totale del circuito di questo elemento equivale a 50 meiri di un filo di rame di un millimetro di diametro, e d'una conduribilità 6,5 rispetio a quella d'un filo di platino di un certo diametro. Una corrente idro-elettrira prodorta da una pila ordinaria di 12 elementi, dà la stessa deviazione di $16 .^{n}$ allorche la lunghezza totale del circuito è di 180 metri. Il diametro del filo di rame è un millimetro e la sua conducibiliı̀ 6.5 : il diametro del filo di platino è 144 millesimi di millimetro, ed uno la sua conducibilita. $\mathbf{E}$ facile di vedere che un millimetro di filo di platino equirale a 313 millimetri di filo di rame, e che per conseguenza 180 metri equivalgono a 56340 metri: cosi il circuito termo-elettrico essendo 50 metri e l'idro-elettrico 36340 metri dello stesso filo, ne viene che la sorgente idro. eletirica vale 1127 volte la sorgente termo-elettrica. Vedesi egualmente da cio, che una slessa sorgente eleltrica puo dare quantita d'elellricità assai diverse, e quindi correnti diversamente intense, essendo sempre queste quantiti in ragione inversa dei circuiti che le correnti devono traversare, supponendo di riferire queste lungtezze ad una stessa unilà 0 ad uno stesso filo.

Questi principii sono pur sulficenti a comprendere in che consista la tensione di una pila, e come col diverso numero delle sue copie possa aversi una comente diversamente intensa, o la stessa secondo la resistenza, e quindi la sezione, la conducibilità e la lunghezza del cirruito interpolare .

Parlando della conducibilita dei liquidi, Pouillet discorre di una differenza che si riscontra in questo elemento, senza che si faccia carıgiare nè la lunghezza, nè la sezione, nè la natura del liquido e rhe raria serondo la natura relativa del metallo e del liquido. Si si che l'intensita della corrente è diminuita 


\section{?'ו}

allorchè una lamina melallira separa come un diaframma uu liquido in due cavita. Quesia diminuzione è massima col platino e l'oro; ed è la minore allorchè il metallo è attaccato facilmente. Questo elemento, rhe deve comporsi nel suo effetio sulla corrente, e delle polarità secondarie, e di una vera resistenza dovuta al cangiamento di conduttore, è di natura assai difficile e non può di certo introdursi nelle formule di Olın come elemento calcolabile ben conosciuto in tutti $i$ casi,

Termineremo questo breve articolo sulla teoria di $\mathrm{Ohm} \mathrm{di}$ cendo che a torto si è creduto che le conseguenze di questa teoria, conducessero necessariamente ad ammettere la forza elettro-motrice di Volta. Ohm ha adottata questa ipotesi e nella sua formola $I=\frac{E}{R}$, E rappresenta la forza elettromotrice. Noi abbiamo però visto che tutte le conseguenze risultanti dalle leggi della resistenza, sono affatıo indipendenti da quesin termine $E$, che si prende e si puo prender per costante, ariche in pratica, oggi che abbiamo pile termo-elettriche o idroelettriche a forza costante.

Nulla s'oppone ad introdurre in lungo di $E$, una espressione che si appoggi sulla teoria chimica. Il metallo positivo ha affinità per uno degli elementi del liquido rhe si scompone; questo elemento ha affinità coll'altro con cui è combinato; e questa affinità è contraria a quella del metallo pel primo. E certo ancora che queste affinità variano serundo la temperatura, secondo la quantità d'acido del liquido, che tende a combinarsi all' ossido formato . - Non ardirei, come ha fatto Daniell, di indicare colle letlere $a, b, c$ queste affinita favorevoli e contrarie alla produzione della corrente. giacchè la formola non avrebbe significato alcuno. 'Tocra all' esperienza a parlare : si concepisce però la possibilitì di confermare anche con queste viste la teoria chimica; e una volta che questo fosse fatto, si vede la possibilita di pervenire a determinare i rapporti delle diverse affinita, deducendoli dalle varie intensita delle correnti generale. 